known UV problems that require renormalization. However, in LQG, background independence in fact assures that this limit exits without any UV divergences. For finite value of the $\epsilon$ the extra loop created by the quantum constraint can entangle the weave of links and nodes in the given spin network around the intersection (first three diagrams on the l.h.s. of Fig. 4). As $\epsilon$ becomes smaller the added loop shrinks and there is a critical value $\epsilon_{c}$ after which it can no longer wind around any of the neighboring links. At this point changing the value of $\epsilon$ amounts to a trivial deformation of the extra loop that, according to the combinatorial nature of the quantum states of gravity, has no physical effect. Therefore, for sufficiently small $\epsilon$ the action of the regulated constraint becomes regulator independent and the limit is defined without need of renormalization (see Fig. 4). This result also holds when coupling gravity with the matter of the standard model; the combinatorial nature of the states of quantum gravity provides a physical regulator for all interactions.

\section{Perspectives and Conclusions}

We have discussed how the dynamical equation of quantum gravity can be promoted to a quantum operator, and how the dynamics of the theory is in the solutions of the quantum Hamiltonian constraint. Although many solutions to the equation are known, there is no complete control of the space of solutions at present. A systematic approach to investigate the solution space is the path integral representation which in the case of LQG is known as the spin foam approach. In it, physical transition amplitudes are computed as sums of amplitudes associated with histories of spin network states (Fig. 5). These histories can be interpreted as the quantum counterpart of space-time: they represent the quantum evolution of the quantum states of space geometry.

Despite the fact that a full understanding of the dynamics of LQG has not yet been achieved, there are interesting physical situations where one can bypass these limitations. One of these is the computation of black hole entropy briefly mentioned above and the other is the application of the framework to systems with additional symmetry. Important examples are the study of quantum cosmology and the near-singularity regime in black hole physics, where due to symmetry assumptions most of the technical problems of the full theory can be overcome. Even though these symmetry-reduced models must be regarded as toy models, as an infinite number of degrees of freedom are ignored in the treatment, there are interesting results that indicate that the singularity problem of classical general relativity would be resolved due to the fundamental discreteness predicted by LQG.

Another important open issue in LQG is the semiclassical limit: how to recover from the fundamental polymer-like excitations of LQG the smooth physics of general relativity and the standard model at low energies.

LQG realizes a unification between the principles of general covariance and those of quantum mechanics. The approach suggests that the outstanding problem of divergences in QFT's and singularities in classical general relativity are resolved when the quantum dynamical degrees of freedom of gravity are included in a background independent manner. The results obtained so far are encouraging; future research will tell whether all this is consistent with the so far elusive nature of quantum gravity..

\section{About the author}

Alejandro Perez is Maitre de Conference HDR at l'Universite AixMarseille II, member of the quantum gravity group at the Centre de Physique Théorique de Marseille. His research focuses mainly on background independent approaches to quantum gravity, loop quantum gravity and spin foams. He has also worked on various aspects of classical general relativity.

\section{Reference}

[1] C. Rovelli, Quantum Gravity, Cambridge Univ. Press, (2005).

\title{
Phussics in daily life: seeing under water
}

\section{L.J.F. (Jo) Hermans,}

Leiden University • The Netherlands

M ost physicists realize that the human eye is not made for seeing under water. For one thing, if we open our eyes under water to see what's going on, our vision is blurred. The reason is obvious: since the index of refraction of the inner eye is practically that of water, we miss the refractive power of the strongly curved cornea surface. With its $1 / f$ of about 40 diopters it forms an even stronger lens than the actual eye lens itself. Could we repair that with positive lenses? In view of the strong curvature of the cornea surface (radius $8 \mathrm{~mm}$ ), the idea of replacing it by a glass lens in a water environment is beyond hope. We really need to restore the air-water interface in front of the cornea, and that is precisely what our diving mask does.

But there is more to it: under water, our field of vision is reduced dramatically. Whereas we normally have a field of more than $180^{\circ}$ due to the refraction at the air-cornea

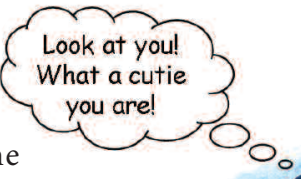

interface, we loose that benefit once we're under water. The diving mask does not repair that, as schematically indicated in the figure.

So, scuba divers, beware! You have to turn your head much further than you may think necessary, if you want to be sure that you are not followed by a shark. -
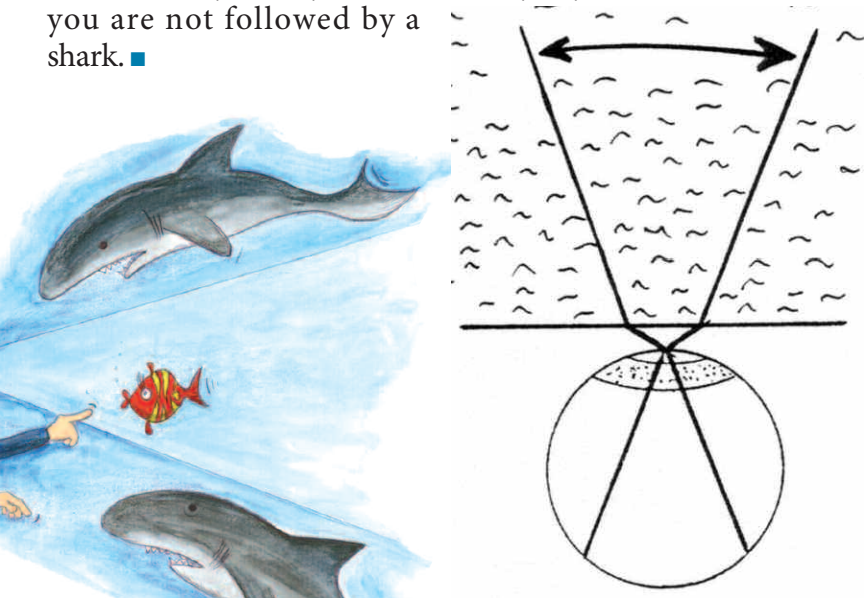